\title{
Propuesta de un índice de seguridad de cruces peatonales para la Ciudad de México
}

\author{
Ruth Pérez López, ${ }^{1}$ Jorge Alberto Montejano Escamilla, ${ }^{2}$ Camilo Alberto Caudillo \\ Cos, ${ }^{2}$ Luz Yazmín Viramontes Fabela, ${ }^{3}$ Tonatiuh Suárez Meaney, ${ }^{4}$ Amilcar Morales \\ Gama, ${ }^{2}$ Mario Ledesma Arreola ${ }^{2}$ y María de los Dolores Sánchez Castañeda ${ }^{5}$
}

Forma de citar Pérez López R, Montejano Escamilla JA, Caudillo Cos CA, Viramontes Fabela, LY, Suárez Meaney T, Morales Gama A, et al. Propuesta de un índice de seguridad de cruces peatonales para la Ciudad de México. Rev Panam Salud Publica. 2019;43:e6. https://doi.org/10.26633/RPSP.2019.6

RESUMEN Objetivo. Proponer un indice de seguridad de cruces peatonales (ISCP) sobre vialidades primarias en Ciudad de México para calificar los cruceros peatonales semaforizados, y contrastar el ISCP con hechos de tránsito para probar, en forma empírica, si hay alguna asociación entre la calidad de los cruceros y la siniestralidad.

Métodos. Identificación de los criterios del indice mediante una revisión del estado del arte, ponderación de los criterios para generar el ISCP mediante el método de análisis multicriterio, diseño de una muestra aleatoria estratificada de cruces peatonales $(n=490)$ y su evaluación.

Resultados. Relativo a la evaluación de los cruceros mediante el ISCP, destaca que 91,3\% de los cruces evaluados en Ciudad de México no cuentan con las condiciones óptimas para resguardar la seguridad de los peatones, con el macrocriterio "Accesibilidad" como el peor calificado. En lo referente al modelaje, resalta que tanto la mezcla de usos del suelo como la distancia de cruce son las variables explicativas más importantes para predecir hechos de tránsito.

Conclusiones. El análisis mostró con relativo éxito la relación entre algunas de las variables (criterios) que conforman el ISCP con los hechos de tránsito. En muchos casos, esto muestra coherencia teórica. En otros, abre preguntas de investigación.

Palabras clave Accidentes de tránsito; prevención de accidentes; peatones; México.

Consejo Nacional de Ciencia y Tecnología, Ciudad de México. Enviar correspondencia a Ruth Pérez López, rperezl@conacyt.mx

2 Centro de Investigación en Ciencias de Información Geoespacial, Ciudad de México, México.

Centro de Estudios de Movilidad Peatonal A.C., Ciudad de México, México.

4 Instituto de Geografía de la Universidad Nacional Autónoma de México, Ciudad de México, México.

5 Instituto Tecnológico Autónomo de México, Ciudad de México, México.
En México, los accidentes de tránsito constituyen un problema importante de salud pública al ser la primera causa de muerte en niños y jóvenes de 1 a 24 años de edad (1). Tan solo en 2016, se registraron 16185 personas fallecidas por hechos de tránsito $(2)^{6}$. A pesar de que en zonas

\footnotetext{
En este artículo se utilizan de manera indistinta los conceptos de hecho vial/de tránsito, accidente $\mathrm{vial} /$ de tránsito y siniestro vial.
}

urbanas y suburbanas las colisiones de vehículos con peatones representan tan solo 3,7\% del total de los hechos de tránsito, los transeúntes contabilizan $23,5 \%$ de las víctimas fatales en el nivel nacional y $57,7 \%$ en Ciudad de México (3). Según datos de la Secretaría de Seguridad Pública (SSP), 61\% de los atropellamientos en Ciudad de México ocurre en ejes viales, avenidas principales y vías de acceso controlado aun cuando estas 
representan solo $11 \%$ de la red vial total (4). Además, debido a las mayores velocidades sobre estas vialidades, el riesgo de morir atropellado es más alto (5).

Por lo anterior, el presente trabajo tiene por objetivo proponer un índice de seguridad de cruces peatonales (ISCP) sobre vialidades primarias en México para evaluar las condiciones de cruce y contrastar dicho índice con hechos de tránsito para probar, en forma empírica, si existe asociación entre la calidad de los cruceros y los atropellamientos. A pesar de la existencia de diferentes índices con estas características en el nivel internacional, México no cuenta con un índice de esta naturaleza. La falta de homologación de la normativa en materia de diseño de cruces peatonales reflejada en los programas "Cruces Seguros" y "Pasos Seguros" llevados a cabo en época reciente (20142016) en Ciudad de México por la Agencia de Gestión Urbana (AGU) y la Autoridad del Espacio Público (AEP), respectivamente, nos muestra la urgencia de estandarizar los criterios de diseño de cruces. A pesar de este vacío, se reconoce el interés creciente del gobierno local por el tema de la seguridad vial. La Ley de Movilidad (6) y el Programa Integral de Movilidad (7) publicados en 2014, el nuevo reglamento de tránsito que establece nuevos límites de velocidad (8), la estrategia "Visión 0 Accidentes" adoptada por el gobierno a principios de 2016, y el Programa Integral de Seguridad Vial de 2017 (9) nos ponen frente a un contexto favorable para el uso aplicado de esta investigación. El índice que se propone a continuación pretende ser una herramienta práctica y de aplicación sencilla que pueda convertirse en un instrumento útil para las autoridades. Mediante la aplicación de este instrumento y la identificación de los criterios calificados como negativos, las autoridades serán capaces de detectar las acciones y obras requeridas específicamente para cada cruce para mejorar su accesibilidad y seguridad.

\section{MATERIALES Y MÉTODOS}

En la actualidad, existen dos grandes tendencias metodológicas para la generación de índices de seguridad en cruces peatonales: la que parte de la observación, conocimiento experto y se sirve de la estadística descriptiva (10-12), y la que toma como base el conocimiento experto y lo modela mediante técnicas de análisis multicriterio (13-17). En época reciente, se observa también una corriente que plantea un modelado no lineal basado solo en una variable independiente (14). Por otra parte, existen dos tendencias conceptuales para aproximarse a la problemática: el análisis de los hechos de tránsito (13) y las que, además, analizan los posibles hechos de tránsito $(11,17)$. A pesar de sus diferencias de aproximación, todas las corrientes coinciden en que se deben generar índices de seguridad sin partir de datos de accidentalidad dado que, en general, existe un problema con la consistencia y calidad de los datos de accidentes, y porque en muchas ocasiones los accidentes no ocurren en forma iterativa en un mismo cruce $(13,18)$. La gran mayoría de las investigaciones consultadas evalúan una poca cantidad de cruces, en contraste con la amplia muestra analizada en el presente trabajo.

Con el punto de partida en el marco metodológico del trabajo de Basile et al. (13), se desarrolló este trabajo en seis fases: a) identificación, jerarquización y selección de los criterio, b) determinación de los rangos de valor de los criterios y su ponderación mediante la técnica AHP (por sus siglas en inglés de analytic hierarchic process), c) diseño de la muestra, d) levantamiento de datos, e) cálculo del ISCP y f) confrontación del ISCP con datos de atropellamientos de SSP-2011-2016.

\section{Identificación, jerarquización y selección de los criterios}

Para seleccionar la lista de criterios, se realizó una revisión del estado del arte (53 documentos especializados) que permitió identificar 94 variables referentes a la seguridad, la comodidad y la calidad de los cruces peatonales y de su entorno inmediato. Estas variables fueron luego agrupadas y ordenadas de mayor a menor frecuencia de citación. Para complementar el análisis documental, se utilizaron los resultados de una encuesta efectuada con anterioridad a 1000 peatones, en la cual exploró su sentimiento de seguridad al cruzar la calle y las principales dificultades a las que se enfrentan en este proceso (19). Después de una retroalimentación de los criterios durante un taller de trabajo en el marco de un congreso especializado (20), se depuró la lista y se decidió validarla con un número final de 19 criterios ordenados en cinco macrocriterios: accesibilidad, visibilidad, diseño, señalamiento horizontal y semaforización (cuadro 1 y figura 1).

\section{Determinación de rangos de valor y ponderación de criterios}

Los rangos de valor de cada criterio emanaron de índices internacionales, del Manual Técnico de Accesibilidad de la Secretaría de Desarrollo Urbano y Vivienda (SEDUVI) de México (21) y de la opinión de expertos. Todos los valores se normalizaron para variar entre 0 y 1 , con los valores cercanos a 1 como positivos (cuadro 1).

Para la ponderación de los criterios y macrocriterios se utilizó el método AHP (por sus siglas en inglés de analityc hierarchy process), el cual se define como una aproximación a la toma de decisiones multiatributo basadas en el concepto de comparaciones por pares (pairwise comparisons) (22). En este modelo se plantearon tres niveles jerárquicos verticales (figura 1). De acuerdo con el método $\mathrm{AHP}$, en caso de una estructura jerárquica con niveles definidos por $\mathrm{J}$ criterios, $\mathrm{M}$ macrocriterios y una meta, es necesario evaluar el peso de los valores asociados a un macrocriterio, y el peso general del macrocriterio que contribuye a la meta general (el índice de seguridad).

Así, el ISCP estará determinado por la siguiente ecuación:

$$
\begin{aligned}
& \text { ISCP }=\sum_{m} w_{m} \cdot C_{m} \\
& C_{m}=\sum_{j}\left(w_{j}^{m} \cdot A_{j}\right)
\end{aligned}
$$

Donde:

$w_{j}^{m}$ es el peso del criterio general de $A_{j}$ asociado a un macro criteriogeneral $C_{m} \mathrm{y}$

$w_{m}$ es el peso del macro criterio general $C_{m}$ que contribuye a la meta general ISCP (13).

La ponderación de cada criterio se realiza con base en la escala semántica de valor propuesta por Saaty (23) que varía del 1 al 9, comparando todos los criterios entre ellos. Para la aplicación del método, se invitaron a diez expertos en movilidad y seguridad vial. Cada participante llevó a cabo el ejercicio a través de una plataforma AHP en línea (24). Los resultados de la ponderación se muestran entre paréntesis en la figura 1.

\section{Diseño de la muestra}

Puesto que el índice de seguridad peatonal construido no es una medida de estimación estadística, no se requería un 
CUADRO 1. Criterios seleccionados y rangos de valor

\begin{tabular}{lr}
\hline \multicolumn{1}{c}{ Accesibilidad } & \\
\hline Señal audible y/o vibratoria & 1 \\
Existe y está en funcionamiento & 0 \\
No está en funcionamiento & 0 \\
No existe & 1 \\
Pavimento táctil & 0 \\
Existe en ambas esquinas & \\
No existe en alguna de las esquinas & 1 \\
Calidad de la banqueta en área de espera peatonal & 0,5 \\
Buena calidad del pavimento en ambas esquinas (superficie lisa) & 0 \\
Calidad del pavimento regular en alguna de las esquinas (grietas) & 0 \\
Mala calidad del pavimento en alguna de las esquinas (hoyos y cambios de nivel) & 1 \\
No hay banqueta en el área de espera peatonal & 0,5 \\
Calidad del pavimento en área de cruce peatonal & 0 \\
Buena calidad del pavimento (superficie lisa) & \\
Calidad del pavimento regular (grietas) & 1 \\
Mala calidad del pavimento (hoyos y cambios de nivel) & 0,5 \\
Obstáculos fijos & 0 \\
Cruce sin obstáculos fijos (en ambas esquinas y sobre el cruce) & \\
Cruce con un obstáculo fijo & 1 \\
Cruce con más de un obstáculo fijo & 0 \\
Obstáculos móviles & \\
Cruce sin obstáculos móviles (en ambas esquinas, sobre el cruce y en isleta) & \\
Cruce con uno o más obstáculos móviles &
\end{tabular}

\begin{tabular}{lr}
\hline \multicolumn{1}{c}{ Visibilidad } \\
\hline lluminación nocturna & 1 \\
Buena condición de iluminación (hay luminarias en ambos lados del cruce) & 0,5 \\
Condición de iluminación regular (solo una luminaria o luz insuficiente) & 0 \\
Mala o nula condición de iluminación (no hay luminarias sobre el cruce) & 1 \\
Obstáculos que obstruyen la vista & 0 \\
No hay obstáculos fijos o móviles que impiden ver las señales y los vehículos & \\
Hay obstáculos fijos o móviles que impiden ver las señales y/o los vehículos
\end{tabular}

$$
\text { Diseño }
$$

\section{Continuidad de pavimento}

Escalón en una 0 más esquinas 0 en isleta 0 camellón (de más de $5 \mathrm{~mm}$ )

Rampas empinadas y/o angostas (ambas esquinas e isleta 0 camellón)

Rampas rectas, con abanico o alabeo en ambas esquinas, con isleta o camellón (alineadas)

\section{Distancia de cruce}

Tres carriles o menos

Más de tres carriles, con isleta o camellón (no más de tres carriles de cada lado)

Más de tres carriles, sin isleta o camellón (más de tres carriles en un lado)

\section{Carril de contraflujo o doble sentido}

Vialidad de un solo sentido

Vialidad con doble sentido con isleta o camellón

Vialidad con doble sentido sin isleta o camellón

Vialidad con carril de contraflujo con isleta o camellón

Vialidad con carril de contraflujo sin isleta o camellón

\section{Señalamiento horizontal}

\section{Paso de cebra}

Está bien marcado y se ve

No se ve bien y hay falta de mantenimiento

No está marcado en parte 0 en su totalidad

\section{Raya de alto}

Está bien marcada y se ve o no aplica

No se ve bien y hay falta de mantenimiento

No está marcada esquema de muestreo formal con cálculo de tamaño de muestra para alcanzar cierto nivel de precisión o error en las mediciones. Sin embargo, la selección de la muestra se realizó de manera probabilística con el objeto de levantar información de un número balanceado de cruces clasificados en estratos de acuerdo con las frecuencias de atropellamientos en un radio específico en torno a los cruceros. Como marco muestral de cruces a evaluar, se obtuvo una base de datos de atropellamientos de la Secretaría de Seguridad Pública (SSP) 2011-2016, la cual se georreferenció mediante Google API ${ }^{\circledR}$ (18 296 eventos) y se agregó a una base de datos espacial antes integrada de semáforos en vialidades primarias. Los atropellamientos se agregaron en un radio de $20 \mathrm{~m}$ de distancia de cada semáforo. En total, hay 7695 cruces con un radio asociado de $20 \mathrm{~m}$.

La estratificación es una técnica muy utilizada en muestreo, ya que produce muestras representativas y estimadores con varianza pequeña, además de ser un método adecuado cuando las poblaciones son sesgadas (25). El método de construcción de estratos de Dalenius y Hodges es común cuando se requiere realizar estratificaciones univariadas de mínima varianza, donde están representados incluso los cruces que no reportan atropellamientos en el radio asociado (26). El tamaño de muestra total se determinó con base en el presupuesto para el levantamiento de campo. Con base en una tasa de no respuesta de $9,0 \%$, resultaron 550 cruceros a evaluar (de un universo de 7 695). Si el índice de seguridad peatonal se estimara estadísticamente, con este tamaño de muestra y un nivel de confianza del $95 \%$, se esperaría un error de medición de 0,04.

\section{Levantamiento de datos y cálculo del ISCP}

Antes del levantamiento en campo, se realizó la verificación visual, mediante Google Maps ${ }^{\circledR}$, la existencia de los semáforos, con una muestra final de $\mathrm{n}=503$. Luego se georreferenciaron, en forma manual, los 503 puntos exactamente a la mitad del cruce peatonal más cercano, de tal modo que los equipos en campo realizaran una evaluación precisa de las condiciones de un cruce específico. Como ayuda al levantamiento, se programó una aplicación en tabletas digitales con conectividad de red en la que los diversos 
CUADRO 1. Criterios seleccionados y rangos de valor (continúa)

\begin{tabular}{lr}
\hline \multicolumn{2}{c}{ Señalamiento horizontal } \\
\hline Flechas de dirección & 1 \\
Están bien marcadas y se ven & 0,5 \\
No se ven bien y faltan de mantenimiento & 0 \\
No están marcadas & 1 \\
\hline \multicolumn{1}{c}{ Semaforización } \\
\hline Tiempo de espera antes de cruzar & 0 \\
Duración del verde vehicular $\leq 60 \mathrm{~s}$ & \\
Duración del verde vehicular >60 s & 0 \\
Tiempo suficiente de cruce & 0 \\
No aplica (no funciona el semáforo vehicular) & 1 \\
TFS $\leq$ TC & 1 \\
TFS > TC & 1 \\
Vuelta a la derecha o a la izquierda & 0 \\
No aplica & \\
Vuelta a la derecha o a la izquierda con control semafórico & 1 \\
Vuelta a la derecha o a la izquierda sin control semafórico & 0 \\
Semáforo vehicular & \\
Está en funcionamiento & \\
No está en funcionamiento & 1 \\
Semáforo peatonal & \\
Existe y está en funcionamiento en la ubicación correcta (ambos lados) & 0 \\
No está en funcionamiento o está en la ubicación incorrecta (un lado o más) & 0 \\
No existe o solo está puesto en un lado del cruce & \\
\hline
\end{tabular}

TFS, tiempo de fase semafórica peatonal; $D$, distancia de cruce; VP, velocidad promedio del peatón $(0,7 \mathrm{~m} / \mathrm{s})$; TC, tiempo de cruce peatonal.

Fuente: elaboración propia.

equipos (diurnos y nocturnos) calificaban y fotografiaban cada uno de los 19 criterios para los 503 cruces evaluados. Ello permitió la generación de una base de datos recopilada en tiempo real en un servidor y con la cual se obtuvo, en forma automática, el ISCP para cada cruce.

\section{Confrontación del ISCP con datos de atropellamientos de SSP (2011-2016)}

Con el objetivo de explorar la posibilidad de utilizar los datos provenientes del levantamiento en campo para predecir hechos de tránsito - y que en su conjunto forman el ISCP-, se procedió a confrontar primero el valor del ISCP de cada crucero con hechos de tránsito y, luego, a buscar asociaciones entre los valores de los criterios de cada cruce con los hechos de tránsito reportados por la SSP en el período 2011-2016.

Dado que algunos de los 503 cruces tenían valores erróneos en las variables "distancia" y "tiempo de cruce" al momento del levantamiento, estos fueron excluidos del análisis, con lo que quedó una muestra final de 490 cruces.

La variable que se construyó para probar los resultados de campo mediante correlaciones bivariadas fue la cantidad de hechos de tránsito ocurridos a distintas distancias del centroide de cada crucero evaluado (25, 100, 200 y 300 metros). De este primer análisis, se resalta que existe una correlación positiva muy débil entre la calificación de los cruceros (ISCP) con la medida de incidentes $(0,06)$. Incluso, una mejor calificación del crucero se asocia con más incidentes, un resultado opuesto a lo esperado. Dado este resultado contraintuitivo, se optó por probar si los criterios del índice por separado se asociaban con la cuenta de hechos de tránsito. Por ello, se exploraron dos métodos de modelaje.

Por una parte, los modelos inflados por cero son adecuados cuando hay una sobrerrepresentación de ceros y parten de la mezcla de dos modelos que combinan el punto de masa en el cero con una distribución de cuentas propiamente especificada. Los dos modelos combinados son uno de distribución binomial negativa para las cuentas y otro con una distribución binomial (logit) para el componente cero inflado $(27,28)^{7}$. Por

\footnotetext{
Se contrastaron diversos modelos de cuentas: Poisson, Poisson cero inflado y binomial negativo cero inflado. Los últimos fueron elegidos bajo criterios de la bondad de ajuste de los modelos.
}

otro, los árboles de decisión (fast frugal trees), funcionan mediante la clasificación de la variable objetivo en dos grupos y la identificación iterativa de qué variables explicativas y qué cortes son los que producen las mejores predicciones (29).

\section{RESULTADOS}

En lo referente a la calificación de los cruceros evaluados con el ISCP, se resalta que $91,3 \%$ de los cruces no cuentan con las condiciones óptimas para resguardar la seguridad de los peatones, con el macrocriterio de accesibilidad como el peor evaluado $(0,29$ de 1$)$.

Relativo al modelaje, se ajustó un modelo de referencia solo con variables (criterios) de los componentes del ISCP. De las 19 variables del índice, se eliminaron algunas por ser constantes y otras porque no se rechazó la hipótesis nula de que los coeficientes tienen un efecto distinto de cero. Una segunda especificación del modelo incorpora variables del entorno urbano que influyen en la ocurrencia de accidentes, como un índice de mezcla de usos de suelo (entropía), cuya idea subyacente es que a mayor mezcla de uso de suelo habrá mayor diversidad de actividades $y$, por ende, más personas y mayor posibilidad de hechos de tránsito (30). Para su cálculo, se combinaron datos censales y del Directorio Estadístico Nacional de Unidades Económicas 2012 (31). Las clases de usos son habitacional, comercial, ocio y equipamiento urbano. El índice varía de cero a uno: cero significa que el área es monofuncional y uno que hay un equilibrio total entre los usos. El resto de las variables del entorno son diversas medidas de los flujos y velocidades de los vehículos en los cruceros evaluados provistas por la empresa Sin Tráfico. A continuación, se presentan los estadísticos descriptivos de las variables reportadas en los modelos (cuadro 2).

En el modelo de referencia (modelo 1, cuadro 3), cinco variables del ISCP resultaron tener una asociación significativa con los hechos de tránsito, donde tres de ellas tienen el signo esperado. Las primeras dos pertenecen al macrocriterio de accesibilidad: tanto mejor "calidad del pavimento" como mayor "ausencia de obstáculos móviles" se asocian con menos incidentes; en cambio, a mayor "distancia de cruce" se reportan más incidentes. Este factor de diseño puede 
FIGURA 1. Esquema del método AHP y ponderación de criterios con base en este modelado

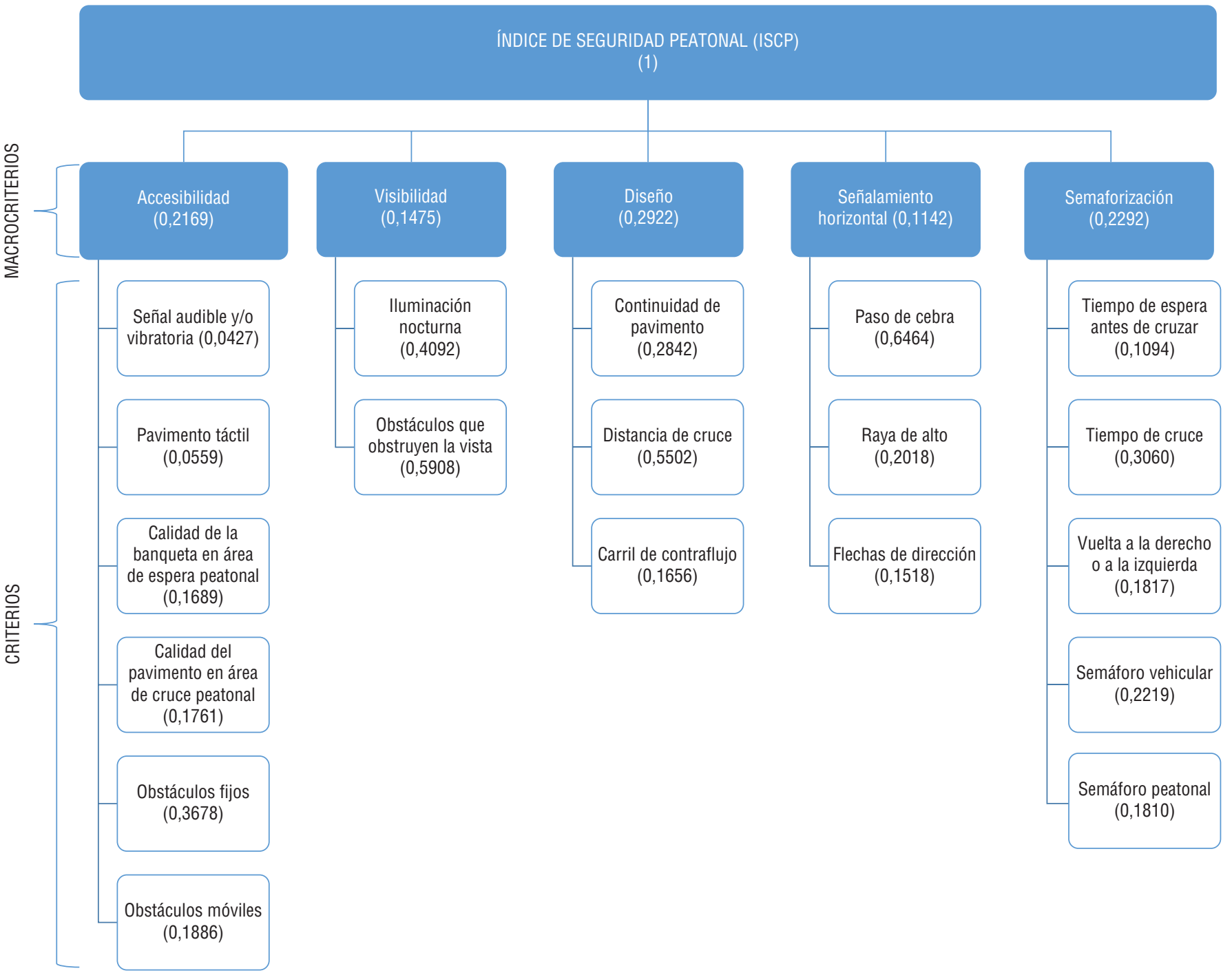

Fuente: elaboración propia con base en (13). Entre paréntesis aparecen los pesos finales de cada criterio, macrocriterio y el índice final.

ser intervenido mediante la reducción de carriles en los cruceros, de modo que se acorte la distancia a cubrir por los peatones. De las variables con signo contrario a lo esperado, la primera, cuando la "continuidad del pavimento" presenta una "rampa adecuada" hay en promedio más incidentes que cuando existe un escalón. Por otra parte, la presencia de la "raya de alto" se asocia de manera positiva con más incidentes. Este resultado contrario a lo esperado no refleja el hecho de que los automovilistas respeten dicho elemento, por lo que su presencia es un componente del señalamiento horizontal importante que debe reforzarse de algún modo para asegurar su función: separar a los transeúntes de los autos.
$\mathrm{Al}$ incorporar las variables del entorno (modelo 2, cuadro 3), el ajuste del modelo mejora de manera sustancial. Las mismas tres variables del ISCP con el signo esperado aún son significativas, pero la variable "raya de alto" deja de serlo y se identifica una asociación positiva marginal entre la ausencia de "obstáculos visuales" y la cantidad de incidentes. Una mayor actividad se relaciona con mayor mezcla de usos del suelo, ya que hay equipamientos que atraen y concentran más personas y vehículos. También la variable del primer quintil promedio del flujo vehicular es significativa y con el signo esperado. Ello implica que una mayor cantidad de automóviles se asocia con más incidentes.

Relativo al modelaje con base en árboles de decisión (32), se clasificaron los cruceros en dos categorías: cruceros de alto riesgo y de bajo riesgo. Se escogió una división para el conjunto de entrenamiento de $70 \%$ y el $30 \%$ restante para prueba (figura 2). El modelo sin variables del entorno, incorpora solo dos de las 19 variables y predice en forma correcta $69,8 \%$ de los cruceros de alto riesgo y $45,7 \%$ de los de bajo riesgo con una precisión total del $54,4 \%$. Al incorporar las variables del entorno, el modelo pone en primer lugar la mezcla de usos de suelo y dos variables más de los criterios del ISCP y mejora el resultado, con 50,9\% de los cruceros de alto riesgo y $70,2 \%$ de los de bajo riesgo correctos. Esto implica que hay una mejora de $3,2 \%$ respecto al puro azar (la precisión es de 63,2\%). Aun con ello, es interesante observar que los re- 
FIGURA 2. Modelo de árbol de decisión

Modelo 1 (sin variables del entorno)

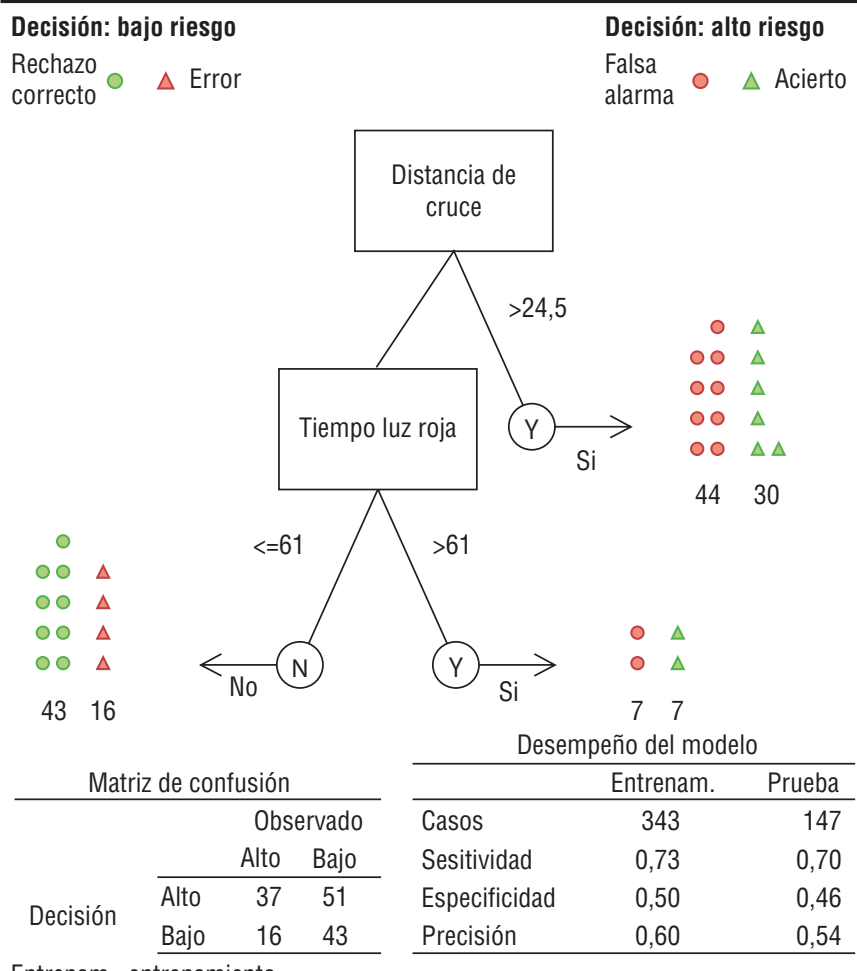

Modelo 2 (con variables del entorno)

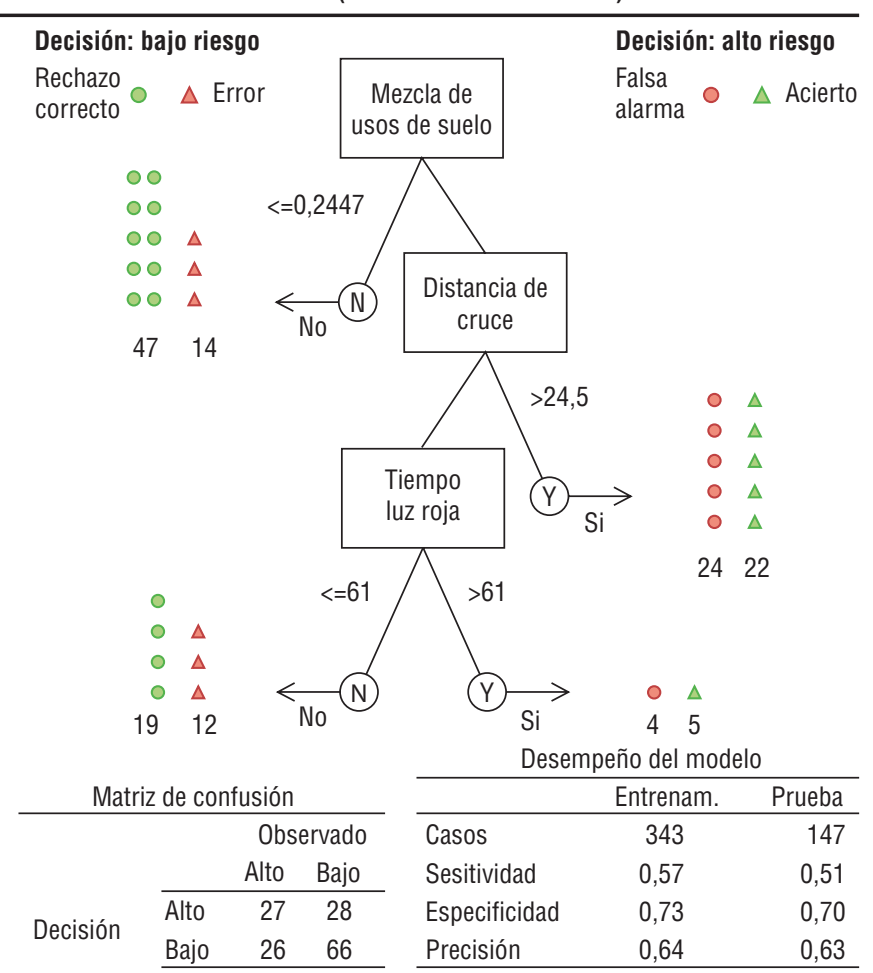

\section{CUADRO 2. Estadísticos descriptivos de las variables en el análisis}

\begin{tabular}{|c|c|c|c|c|c|c|c|}
\hline & Mínimo & Máximo & Media & Desviación estándar & Categoría $0(\%)$ & Categoría $1(\%)$ & Categoría $2(\%)$ \\
\hline ISCP & 0,10 & 0,87 & 0,41 & 0,13 & NC & NC & NC \\
\hline Hechos de tránsito & 0 & 38 & 5,23 & 5,91 & NC & NC & NC \\
\hline Calidad del pavimento en área de cruce peatonal & 0 & 2 & NC & NC & 42 & 35,5 & 22,4 \\
\hline Obstáculos que obstruyen la vista & 0 & 1 & NC & NC & 24,3 & 75,7 & NC \\
\hline Continuidad de pavimento & 0 & 2 & NC & NC & 72,7 & 13,5 & 13,9 \\
\hline Raya de alto & 0 & 2 & NC & NC & 33,1 & 46,1 & 20,8 \\
\hline Distancia del cruce & 6,65 & 227,00 & 27,71 & 15,29 & NC & NC & NC \\
\hline Mezcla de uso de suelo promedio & 0,06 & 0,65 & 0,31 & 0,12 & NC & NC & NC \\
\hline Flujo vehicular (primer quintil promedio) & 0,00 & 3355,25 & 1004,27 & 648,23 & NC & NC & NC \\
\hline
\end{tabular}

ISCP, índice de seguridad de cruces peatonales; NC, no corresponde.

Fuente: elaboración propia.

sultados convergen: la entropía es la variable explicativa más importante, ya que los valores bajos de mezcla de usos de suelo predicen a la clase de bajo riesgo, mientras que en el modelo sin entropía lo es la distancia de cruce (más de 24,5 metros predicen cruceros de alto riesgo).

\section{DISCUSIÓN}

Se resalta que la metodología $\mathrm{AHP}$ es útil para la creación de un ISCP que permita la jerarquización de las condiciones de paso y su posterior mejoramiento infraestructural por parte del gobierno local. Con la finalidad de que la seguridad de los cruces pueda ser incrementada mediante intervenciones puntuales basadas en el mejoramiento de los criterios evaluados de manera negativa, este índice incluye solo criterios de infraestructura, diseño y operación. Dado que este instrumento, en tanto herramienta de política pública busca destacar por su practicidad, se excluyó la medición de variables difícilmente medibles in situ, no cuantificables y/o que dificultan la aplicabilidad del instrumento.

Sin embargo, la subjetividad intrínseca del método AHP parece dificultar su implementación como base para un modelo predictivo. A pesar de que no hacen explícito su uso como método para pronosticar hechos de tránsito (13), y de que no se valida en ese documento la metodología empleada con base en métodos estadísticos, su utilización exitosa en diversos cruceros en Europa pone de relieve las diferencias idiosincráticas que es 
CUADRO 3. Modelos binomial negativo cero inflados

\begin{tabular}{|c|c|c|c|c|}
\hline \multirow{2}{*}{ Variable } & \multicolumn{2}{|c|}{ Modelo $1^{\mathrm{a}}$} & \multicolumn{2}{|c|}{ Modelo $2^{\mathrm{b}}$} \\
\hline & Coeficiente & Error estándar & Coeficiente & Error estándar \\
\hline \multicolumn{5}{|l|}{ Componente de cuentas } \\
\hline Intercepto & 1,546 & $(0,159)^{c}$ & 0,797 & $(0,196)^{c}$ \\
\hline \multicolumn{5}{|c|}{$\begin{array}{l}\text { Calidad del pavimento en área de cruce peatonal } \\
\text { (ref = } 0 \text { "mala") }\end{array}$} \\
\hline Regular & $-0,137$ & $(0,107)$ & $-0,203$ & $(0,104)$ \\
\hline Buena & $-0,222$ & $(0,134)$ & $-0,328$ & $(0,124)^{d}$ \\
\hline Obstáculos móviles (dummy) & $-0,326$ & $(0,101)^{d}$ & $-0,314$ & $(0,098)^{d}$ \\
\hline Obstáculos visuales (dummy) & NC & NC & 0,201 & NC \\
\hline \multicolumn{5}{|c|}{ Continuidad de pavimento (ref = 0 "Hay escalón") } \\
\hline Rampa empinada & 0,207 & $(0,139)$ & 0,224 & $(0,132)$ \\
\hline Rampa adecuada & 0,567 & $(0,142)^{c}$ & 0,538 & $(0,136)^{c}$ \\
\hline \multicolumn{5}{|l|}{ Raya de alto (ref = 0 "No existe") } \\
\hline Falta mantenimiento & 0,247 & $(0,111)^{e}$ & NC & NC \\
\hline Está bien marcada y es visible & 0,117 & $(0,145)$ & NC & NC \\
\hline Distancia de cruce & 0,010 & $(0,004)^{d}$ & 0,009 & $(0,004)^{e}$ \\
\hline Mezcla de usos de suelo & NC & NC & 1,774 & $(0,372)^{c}$ \\
\hline Flujo vehicular (Q1) & NC & NC & 0,0002 & $(0,0001)^{d}$ \\
\hline Log (theta) & 0,328 & $(0,127)^{e}$ & 0,449 & $(0,131)^{c}$ \\
\hline \multicolumn{5}{|l|}{ Componente cero inflado } \\
\hline Intercepto & $-0,869$ & $(0,573)$ & $-1,367$ & $(0,589)^{\mathrm{e}}$ \\
\hline Distancia de cruce & 0,026 & $(0,01)^{d}$ & 0,026 & $(0,01)^{\mathrm{e}}$ \\
\hline Mezcla de usos de suelo & $-6,421$ & $(2,335)^{d}$ & $-4,672$ & $(2,039)^{\mathrm{e}}$ \\
\hline Logaritmo del estadístico de verosimilitud & \multicolumn{2}{|r|}{-1312} & \multicolumn{2}{|c|}{-1293} \\
\hline Criterio de información de Akaike & \multicolumn{2}{|c|}{2649,41} & \multicolumn{2}{|c|}{2613,91} \\
\hline
\end{tabular}

a Incluye solo variables del ISCP.

${ }^{\mathrm{b}}$ Incluye variables del entorno.

${ }^{\mathrm{C}}$ Código de significancia $=0$.

${ }^{\mathrm{C}}$ Código de significancia $=0,001$.

${ }^{\text {e }}$ Código de significancia $=0,05$.

Fuente: elaboración propia.

probable que existan entre los conductores y peatones entre ambos lados del Océano Atlántico (es decir, respeto a los reglamentos de tránsito)

- En esta investigación, se observó cómo el propio ISCP no se correlaciona, por sí mismo, de manera significativa y coherente con el número de atropellamientos observados, incluso presenta un signo contrario al esperado. Ello se puede deber a múltiples factores, comenzando con la condición de subjetividad antes señalada. Además, suponemos que el siguiente factor juega un papel determinante: la falta de precisión del lugar en donde ocurrió el atropellamiento puesto que, por una parte, la SSP registra en forma automática los atropellamientos sobre una intersección vial y no sobre un cruce peatonal y, por otra, reporta en la intersección más cercana los accidentes que ocurren en medio de la vialidad (cuando un peatón se atraviesa por un lugar no señalizado).
- La no consideración del comportamiento del conductor: no se tomó en cuenta la violación del reglamento de tránsito por parte de los conductores que pone en peligro constante a los peatones (al no respetar el alto) y obstaculizan la accesibilidad (al detenerse sobre el paso peatonal).

- Confrontar de manera directa el ISCP con hechos de tránsito y no con una tasa (es decir, número de atropellamientos/número de habitantes y número de trabajadores). ${ }^{8}$

A pesar de lo anterior, ha sido posible modelar con relativo éxito la relación entre algunas de las variables (criterios) que conforman el ISCP con los hechos de tránsito. En muchos casos, esto muestra coherencia teórica. En otros, abre preguntas de investigación.

\footnotetext{
Para los modelos desarrollados sí se generó una tasa, aunque funcionó mejor la cuenta de hechos de tránsito.
}

Si comparamos nuestros resultados con los reportados por Carter et al. (12), podemos observar que parte importante del poder explicativo del índice norteamericano pedestrian intersection safety index (Ped ISI) proviene de la medición de velocidad automotora sobre el cruce evaluado. En nuestro caso también se probaron especificaciones que incluyeron datos de velocidad automotora, tanto para el modelado estadístico como con los árboles de decisión. Sin embargo, solo en los modelos cero inflados encontramos efectos significativos del primer quintil de la velocidad (disponible a solicitud), los modelos presentados aquí muestran solo los flujos vehiculares por su mejor ajuste.

Por otra parte, ambos modelos han mostrado la gran importancia de la mezcla de uso de suelo como factor crucial que puede explicar los hechos de tránsito (en el caso norteamericano, fue el uso comercial del suelo), con ello se corroboraría la importancia de la existencia de flujos para que ocurran hechos de tránsito. Resalta que el modelo desarrollado para Ciudad de México muestra también una asociación positiva y significativa entre distancia de cruce y hechos de tránsito, por lo que el desarrollo de políticas públicas orientadas a reducir el número de carriles podría, en efecto abonar a una menor siniestralidad. Por último, se sugiere que este modelo puede ser mejorado de manera sustancial a través de la detección no solo de los hechos de tránsito, sino también de las situaciones donde se ha registrado potencialmente un hecho de tránsito (conflictos), tal y como la literatura lo sugiere.

Agradecimientos. Los autores agradecen la colaboración del Instituto de Políticas para el Transporte y Desarrollo (ITDP), de la Dirección General de Ingeniería de la Subsecretaría de Control de Tránsito de la Secretaría de Seguridad Pública y de SinTráfico.

Financiación. Esta investigación fue realizada con financiamiento de la Embajada de Francia en México y de la Fundación Mapfre, España.

Conflictos de interés. Ninguno declarado por los autores.

Declaración. Las opiniones expresadas en este manuscrito son responsabilidad del autor y no reflejan necesariamente los criterios ni la política de la RPSP/ PAJPH y/o de la OPS. 


\section{REFERENCIAS}

1. Instituto Nacional de Estadística y Geografía de México. Base de datos de las principales causas de mortalidad en México, 2016. Disponible en: http://www.inegi. org.mx/est/contenidos/proyectos/registros/vitales/mortalidad/tabulados/PC. asp? $\mathrm{t}=14 \& \mathrm{c}=11817$ Acceso el 24 de agosto de 2018.

2. Secretaría de Salud/Secretariado Técnico del Consejo Nacional para la Prevención de Accidentes. Informe sobre la situación de la seguridad vial México 2017. México, 2018. Disponible en: https://drive.google. com/file/d/1IcEIsytf4rET_pZVdQWAbXbAfwccnFA/view Acceso el 24 de agosto de 2018.

3. Instituto Nacional de Estadística y Geografía de México. Base de datos de accidentes de tránsito terrestre en zonas urbanas y suburbanas, 2016. Disponible en: http:// www.inegi.org.mx/est/contenidos/Proyectos/registros/economicas/accidentes/ default.aspx Acceso el 24 de agosto de 2018.

4. Gobierno del Distrito Federal. Pasos seguros. México: Gobierno del Distrito Federal, 2015:15.

5. Richards DC. Relationship between speed and risk of fatal injury: pedestrians and car occupants. Londres: Department for Transport, 2010.

6. Asamblea Legislativa del Distrito Federal. Ley de Movilidad del Distrito Federal. México: Gaceta Oficial del Distrito Federal, número 1899 bis; 14 de julio del 2014. Disponible en: http://data.consejeria. cdmx.gob.mx/portal_old/uploads / gacetas/df335356af9d93c68cac837125d30308. pdf Acceso el 2 de agosto de 2018.

7. Secretaría de Movilidad del Distrito Federal. Programa Integral de Movilidad, 2013-2018. México: Gaceta Oficial del Distrito Federal, número 1965 bis; 15 de octubre de 2014. Disponible en: http://data. consejeria.cdmx.gob.mx/portal_old/ uploads/gacetas/3b3d5d0fb924a67b0b6fa5fdade4adbc.pdf Acceso el 2 de agosto de 2018.

8. Secretaría de Seguridad Pública de México. Reglamento de Tránsito del Distrito Federal. México: Gaceta Oficial del Distrito Federal, número 156 bis; 17 de agosto de 2015. Disponible en: http://www.ssp.df. gob.mx/reglamentodetransito/documentos/nuevo_reglamento_transito.pdf Acceso el 2 de agosto de 2018 .

9. Secretaría de Movilidad de México. Programa Integral de Seguridad Vial, 20162018. México: Gaceta Oficial del Distrito Federal; 2017;59. Disponible en: http:// www.semovi.cdmx.gob.mx/storage/
app/media/PISVI_Low.pdf Acceso el 2 de agosto de 2018 .

10. Cheng W, Zhang N, Li W, Xi J. Modeling and application of pedestrian safety conflict Index at signalized intersections. Discrete Dyn Nat Soc. 2014;2014:1-6.

11. Bian Y, Jian L, Zhao L. Method to determine pedestrians level of service for unsignalized intersections. Appl Mech Mater. 2013;253:1936-43.

12. Carter DL, Hunter WW, Zegeer CV, Stewart JR, Huang HF. Pedestrian and bicyclist intersection safety indices: final report. Washington, DC: Federal Highway Administration; 2006. Disponible en: https://www.fhwa.dot.gov/publications / research / safety / pedbike/06130/06130.pdf Acceso el 28 de agosto de 2018.

13. Basile O, Persia L, Usami DS. A methodology to assess pedestrian crossing safety. Europ Trans Res Rev. 2010;2:129-37.

14. Montella A, Mauriello F. Pedestrian crosswalks safety inspections: safety assessment procedure. Proceedings of 4th International Symposium on Highway Geometric Design 2010;1-17. Disponible en: https://s3.amazonaws.com/academia. edu.documents/7975421/72.pdf?AWSAccess KeyId = A KI A IW OWYYGZ2Y53UL3A\&Expires $=1535134366 \&$ Signature$=0 \mathrm{U} 2 \mathrm{Twp} \% 2 \mathrm{BJ}$ i5qP3le $1 \mathrm{Kh} \mathrm{GgwcH}-$ bA7M\%3D\&response-content-disposition= in 1 in e \% 3 B \% 20 file na m e 3 DPedestrian_Crosswalks_Safety_Inspections.pdf Acceso el 28 de agosto de 2018.

15. Hannah C. A computational model of pedestrian road safety: the long way round is the safe way home. Accident Anal Prev. 2018. En prensa. Disponible en: https:// www.sciencedirect.com/science/article/ pii/S0001457518302185 Acceso el 28 de agosto de 2018.

16. Soilán M, Riveiro B, Sánchez-Rodríguez A, Arias P. Safety assessment on pedestrian crossing environments using MLS data. Accident Anal Prev. 2018;111:328-37.

17. Asadi-shekari Z, Moeinaddini M, Shah MZ. Pedestrian safety index for evaluating street facilities in urban areas. Safety Sci. 2015;74:1-14

18. Cafiso S, García A, Cavarra R, Romero Rojas MA. Pedestrian crossing safety improvements: before and after study using traffic conflict techniques. 4th International Symposium on Highway Geometric Design. 2010:2-5.

19. Pérez López R. Quand le piéton défie la ville: traverser la chaussée à Mexico. Environnement Urbain. 2015;9:1-27.
20. Pérez López R, Viramontes Fabela LY. Taller de construcción de indicadores para evaluar cruces peatonales. II Congreso Internacional de Peatones. Cholula, México. 27 de mayo, 2015.

21. Secretaría de Desarrollo Urbano y Vivienda. Manual Técnico de Accesibilidad México: Secretaría de Desarrollo Urbano y Vivienda: 2007.

22. Malczewski J. GIS and multicriteria decision analysis. Nueva York: J. Wiley \& Sons; 1999.

23. Saaty T. Decision making with the analytic hierarchy process. Int J Serv Sci. 2008;1(1):83-98.

24. Goepel, KD. BPMSG AHP priority calculator. 2018. Disponible en: http://bpmsg. com/ahp-online-calculator/

25. Kareem AO, Oyeyemi GM, Aiyelabegan Adijat B. A note on the efficiency of geographic stratification. International Journal of Mathematics and Statistics Invention. 2015;3(4):1-10.

26. Dalenius T, Hodges, JL. Minimum variance stratification. Journal of the American Statistical Association. 1959;54(285): 88-101.

27. Colin A, Pravin K. Regression Analysis of Count Data. Nueva York: Cambridge Press; 1998.

28. Zeileis A, Kleiber C, Jackman S. Regression Models for Count Data in R. J Stat Soft. 2008;27(8):1-25.

29. International Business Machines Corporation (IBM). Creating Decision Trees. Disponible en: https:/ /www.ibm.com/support/ knowledgecenter/es/SSLVMB_22.0.0/ com.ibm.spss.statistics.help/spss/tree/ idh_idd_treegui_main.htm Acceso el 24 de agosto de 2018.

30. Cervero R, Kockelman K. Travel demand and the 3Ds: density, diversity, and design. Transportation Research Part D. : Transport and Environment. 1997;2(3):199-219.

31. Instituto Nacional de Estadística y Geografía de México. Directorio Estadístico Nacional de Unidades Económicas, 2012. Disponible en: http://www.beta.inegi.org.mx/app/mapa/ denue/ Acceso el 24 de agosto de 2018.

32. Phillips N, Neth H, Gaissmaier W, Woike J. FFTrees: a toolbox to create, visualise, and implement fast-and-frugal decision trees. Judgm Decis Mak. 2017;12(4):344-68.

Manuscrito recibido el 23 de enero de 2018. Aceptado para su publicación, tras revisión, el 30 de agosto de 2018. 
ABSTRACT

Proposed Pedestrian Crosswalk Safety Rating for Mexico City

Keywords
Objective. Propose a pedestrian crosswalk safety rating (PCSR) for primary roads in Mexico City in order to rate crosswalk safety at intersections with a traffic light and then compare the PCSR with traffic accidents so as to empirically determine any association between the quality of the crosswalk and the traffic accident rate.

Methods. Identify criteria for the rating system through a state-of-the art review; weight the criteria to create a rating system through multicriterion analysis; design a stratified random sample of crosswalks $(n=490)$; and evaluate the data set.

Results. Through the PCSR, 91.3\% of the crosswalks evaluated in Mexico City were found not to offer the conditions required to protect pedestrian safety; the "access" macro-criterion received the worst scores. The modelling shows that mixed land use and the length of the crosswalk are the most important variables in predicting traffic accidents.

Conclusions. The analysis was relatively successful in showing the relationship between some variables (criteria) of the PCSR and traffic accidents. In many cases, this shows theoretical coherence; in others, research questions are raised.

Accidents, traffic; accident prevention; pedestrians; Mexico.
RESUMO

\section{Proposta de um índice de segurança de travessia de pedestres para a Cidade do México}

\author{
Palavras-chave
}

Objetivo. Propor um índice de segurança de travessia de pedestres (ISTP) para as principais vias públicas na Cidade do México para classificar as travessias de pedestres semaforizadas e comparar o ISTP com os dados de trânsito para comprovar empiricamente se existe associação entre a qualidade dos locais de travessia e a taxa de acidentes.

Métodos. Foram identificados os critérios do índice com uma revisão do conhecimento atual e os critérios para gerar o ISTP foram ponderados com uso do método de análise de decisão multicritério e delineamento e avaliação de uma amostra aleatória estratificada de travessias de pedestres $(n=490)$.

Resultados. Com respeito à avaliação das travessias com o uso do ISTP, verificou-se que 91,3\% das travessias avaliadas na Cidade do México não têm condições ideais para resguardar a segurança dos pedestres, com o macrocritério "acessibilidade" com a pior qualificação. Quanto à modelagem, observou-se que a mescla de usos do solo e a distância da travessia são as variáveis explicativas mais importantes para predizer a ocorrência de acidentes de trânsito.

Conclusões. A análise demonstrou com relativo sucesso a relação entre algumas variáveis (critérios) que compõem o ISTP e acidentes de trânsito. Houve uma coerência teórica em muitos casos, porém em outros suscitou dúvidas a serem investigadas.

Acidentes de trânsito; prevenção de acidentes; pedestres; México. 